OPEN ACCESS

Edited by:

Arnaud Scherberich,

University Hospital of Basel,

Switzerland

Reviewed by:

Sarah Hosgood,

University of Cambridge,

United Kingdom

Shin Enosawa,

National Center for Child Health and

Development (NCCHD), Japan

*Correspondence:

José Tomás Egaña

jte@uc.cl

Rolando Rebolledo

rarebol॥uc.cl

Specialty section:

This article was submitted to Tissue Engineering and Regenerative

Medicine,

a section of the journal

Frontiers in Bioengineering and

Biotechnology

Received: 16 October 2021 Accepted: 24 November 2021

Published: 15 December 2021

Citation:

Veloso-Giménez V, Escamilla $R$,

Necuñir $D$, Corrales-Orovio $R$

Riveros S, Marino C, Ehrenfeld $C$, Guzmán $C D$, Boric MP, Rebolledo $R$ and Egaña JT (2021) Development of a

Novel Perfusable Solution for ex vivo

Preservation: Towards Photosynthetic

Oxygenation for

Organ Transplantation

Front. Bioeng. Biotechnol. 9:796157.

doi: 10.3389/fbioe.2021.796157

\title{
Development of a Novel Perfusable Solution for ex vivo Preservation: Towards Photosynthetic Oxygenation for Organ Transplantation
}

\begin{abstract}
Valentina Veloso-Giménez ${ }^{1}$, Rosalba Escamilla ${ }^{1,2}$, David Necuñir ${ }^{1}$, Rocío Corrales-Orovio ${ }^{1,3}$, Sergio Riveros ${ }^{4}$, Carlo Marino ${ }^{4}$, Carolina Ehrenfeld ${ }^{1,2}$, Christian Dani Guzmán ${ }^{5}$, Mauricio P. Boric ${ }^{2}$, Rolando Rebolledo ${ }^{1,6 *}$ and José Tomás Egaña ${ }^{1 *}$

${ }^{1}$ Schools of Engineering, Medicine and Biological Sciences, Institute for Biological and Medical Engineering, Pontificia Universidad Católica de Chile, Santiago, Chile, ${ }^{2}$ Department of Physiology, Faculty of Biological Sciences, Pontificia Universidad Católica de Chile, Santiago, Chile, ${ }^{3}$ Division of Hand, Plastic and Aesthetic Surgery, LMU Munich, University Hospital, Munich, Germany, ${ }^{4}$ Department of Digestive Surgery, Faculty of Medicine, Pontificia Universidad Católica de Chile, Santiago, Chile, ${ }^{5}$ Sky-Walkers SpA, Litueche, Chile, ${ }^{6}$ Hepatobiliary and Pancreatic Surgery Unit, Surgery Service, Hospital Dr. Sótero del Río, Santiago, Chile
\end{abstract}

Oxygen is the key molecule for aerobic metabolism, but no animal cells can produce it, creating an extreme dependency on external supply. In contrast, microalgae are photosynthetic microorganisms, therefore, they are able to produce oxygen as plant cells do. As hypoxia is one of the main issues in organ transplantation, especially during preservation, the main goal of this work was to develop the first generation of perfusable photosynthetic solutions, exploring its feasibility for ex vivo organ preservation. Here, the microalgae Chlamydomonas reinhardtii was incorporated in a standard preservation solution, and key aspects such as alterations in cell size, oxygen production and survival were studied. Osmolarity and rheological features of the photosynthetic solution were comparable to human blood. In terms of functionality, the photosynthetic solution proved to be not harmful and to provide sufficient oxygen to support the metabolic requirement of zebrafish larvae and rat kidney slices. Thereafter, isolated porcine kidneys were perfused, and microalgae reached all renal vasculature, without inducing damage. After perfusion and flushing, no signs of tissue damage were detected, and recovered microalgae survived the process. Altogether, this work proposes the use of photosynthetic microorganisms as vascular oxygen factories to generate and deliver oxygen in isolated organs, representing a novel and promising strategy for organ preservation.

Keywords: organ preservation, organ perfusion, ischemia, hypoxia, photosynthetic microorganisms, Chlamydomonas reinhardtii, photosynthesis

\section{INTRODUCTION}

The lack of appropriate tissue oxygenation represents a major issue in several medical areas, being particularly relevant in the transplantation field, where organ ischemia induces hypoxia, limiting their ex vivo preservation time, as well as their further clinical outcome after transplantation (Hosgood et al., 2012; Giwa et al., 2017).

Aiming to decrease the oxygen requirements of isolated organs, static cold storage (SCS) has been the gold standard in clinical transplantation settings, reducing metabolism and therefore oxygen 
consumption in about $90 \%$ (Karangwa et al., 2016). However, this technique is limited in terms of preservation time and for maintaining the integrity of suboptimal grafts derived from expanded criteria donor (ECD) and donation after circulatory death (DCD), which are more sensitive to damage (Soo et al., 2020; Vries et al., 2020). SCS represents the first step in the cascade of ischemia reperfusion injury ensuing organ implantation (Eltzschig and Eckle, 2011), triggering tissue damage through oxidative stress and inflammation (Slegtenhorst et al., 2014). Moreover, it is associated with organ allograft dysfunction and acute rejection, reducing the graft survival (Zhao et al., 2018).

Aiming to provide oxygen ex vivo, novel technologies have been established. Among them, the use of machine perfusion is promising. Some of these systems are based on extracorporeal oxygenation devices, where erythrocytes are oxygenated in a dynamic system that allows them to recirculate through the organ vascular network. Although recent clinical data support their safety and efficacy (Xu et al., 2020; Tatum et al., 2021), the use of blood generates additional complications, because it is not always available on site, has a relative short preservation time and requires the inclusion of a membrane oxygenator, which substantially increases the total cost (Aburawi et al., 2019). Additionally, blood recirculation generates hemolysis, which can be toxic as free hemoglobin can cause inflammation and oxidative stress (Gkoumassi et al., 2012; Bodewes et al., 2021), thus the development of novel perfusable solutions for ex vivo oxygenation is an active field of research. The use of oxygen carriers as an alternative to erythrocytes has been widely studied, and promising results have been described for hemoglobin-based oxygen carriers obtained from annelids (e.g. Arenicola marina) (Bhattacharjee et al., 2020; Bodewes et al., 2021). However, they require intensive purification for their use, and their passive oxygen release kinetics makes them poorly controllable depending on the organ metabolic needs (Le Meur et al., 2020; Varney et al., 2021). As a purely synthetic alternative, perfluorocarbons have limitations due to the difficulty of controlling the kinetics of oxygen release, as well as the complexity of manufacturing and the need to incorporate them into different emulsions, limiting their widespread adoption in organ preservation (Malchesky, 2017; Jägers et al., 2021). As an alternative method for oxygen supply, we and others have proposed that the induction of local photosynthesis could modulate oxygen tension in hypoxic tissues. Based on this, photosynthetic therapies aim to generate a local symbiotic relationship between animal and photosynthetic cells where, in the presence of light, both metabolisms could be coupled with each other (Chávez et al., 2020). This approach has been described by our group in recent in vitro (Hopfner et al., 2014; Centeno-Cerdas et al., 2018; Chávez et al., 2021) and in vivo (Schenck et al., 2015; Chávez et al., 2016) studies, and further confirmed by other independent groups, highlighting its potential application in several medical fields, including tissue engineering and regeneration (Yamaoka et al., 2012; Evron et al., 2015; Haraguchi et al., 2017; Chen et al., 2020), heart ischemia (Cohen et al., 2017), and tumor treatment (Huo et al., 2020; Liu et al., 2020; Qiao et al., 2020). Moreover, an ongoing clinical trial is confirming its safety for tissue regeneration in humans (Obaíd et al. (2021) accepted for publication. ClinicalTrials.gov Identifier: NCT03960164).

Taking all this into consideration, in this work we propose that the development of perfusable photosynthetic solutions could allow organ preservation by in situ vascular oxygenation. To accomplish this long-term goal, here we describe the first generation of a perfusable photosynthetic solution for organ preservation, providing key scientific insights about its therapeutic potential.

\section{MATERIALS AND METHODS}

\subsection{Microalgae Culture}

Cell-wall deficient UVM4-GFP C. reinhardtii strain (cw15-30derived) was kindly provided by Prof. Jörg Nickelsen (LUM, Germany) and cultured as described before (Neupert et al., 2009). Briefly, microalgae were grown photomixotrophically at room temperature $\left(20-25^{\circ} \mathrm{C}\right)$ on either solid Tris Acetate Phosphate (TAP) medium with $1.5 \%(\mathrm{w} / \mathrm{v})$ agar or in bottles containing different volumes of liquid TAP medium placed in an orbital shaker $(180 \mathrm{rpm})$. For light stimulation, a lamp with the full spectrum of white light was used to provide continuous light exposure $\left(30 \mu \mathrm{E} / \mathrm{m}^{2} \mathrm{~s}\right)$ (Harris, 2009). Cell concentration was determined using a Neubauer chamber.

\subsection{Generation of the Photosynthetic Solution for Organ Preservation}

To prepare the PSOP, Ringer's lactate solution was mixed with 0.5\% (w/v) mannitol (RLM; AppliChem Panreac) as impermeant agent (Nicholson and Hosgood, 2017). Liquid cultures of $C$. reinhardtii in exponential growth phase were grown in $100 \mathrm{ml}$ borosilicate glass bottles up to $5 \mathrm{~L}$ depending on the requirements of each experiment. For optimal growth, constant agitation was maintained, either on an orbital shaker or a magnetic stirrer. For harvesting, liquid cultures up to $2 \mathrm{~L}$ were centrifuged and $5 \mathrm{~L}$ cultures were recovered by decantation; thereafter pelleted algae were resuspended in RLM at different cell densities $\left(10^{6}-10^{9} \mathrm{C}\right.$. reinthardtii/ml). As control groups, TAP medium and a mixed solution of TAP:RLM (in 1:1 ratio) were included. For ex vivo kidney perfusion (see below in section 2.10 and section 2.12) and metabolic coupling assay of kidney slices (section 2.8), 5\% (w/v) dextran-70 (H979; AK Scientific Inc) was added to RLM to maintain the oncotic pressure.

\subsection{Microalgae Viability Assays}

After $24 \mathrm{~h}$ of incubation of C. reinhardtii in RLM, TAP or TAP: RLM, viability of the microalgae was determined by examining growth after 5 days of inoculation in agar plates. As viability probe (Jamers et al., 2009), microalgae were diluted to $3 \times 10^{5} \mathrm{C}$. reinhardtii/ml and incubated for $1 \mathrm{~h}$ with $25 \mu \mathrm{M}$ of Fluorescein diacetate (FDA, F1303, Life Technologies). A death control was included by heating C. reinhardtii at $85^{\circ} \mathrm{C}$ for $10 \mathrm{~min}$ and $10^{5}$ events per sample were acquired in BD Influx cell sorter (Becton Dickinson). Data was analyzed with FlowJo software (Becton 
Dickinson) by gating chlorophyll positive cells. In order to discard the contribution of GFP to the FDA signal, unstained, stained, and dead microalgae were used as controls to determine the basal level of fluorescence and set the gate for FDA.

\subsection{Cell Morphology Evaluation}

General morphology of the microalgae in RLM, TAP or TAP: RLM was evaluated by optical microscopy (Leica DM500), as well as by flow cytometry (BD FACSCanto II analyzer, Becton Dickinson). Cell diameter was quantified using 4, 6, 10 and $15 \mu \mathrm{m}$ size marker beads (F13838; Life Technologies), and $10^{5}$ events were recorded in the microalgae gate. Data was analyzed with FlowJo software (Becton Dickinson) by gating chlorophyll positive cells.

\subsection{Oxygen Production of PSOP}

After 0 and $24 \mathrm{~h}$ of incubation in RLM, the oxygen production of PSOP containing different cell densities $\left(0,10^{6}, 10^{7}, 10^{8}\right.$ and $10^{9}$ C. reinhardtii $/ \mathrm{ml}$ ) was measured at $28^{\circ} \mathrm{C}$ using an Oxygraph System (Hansatech Instruments). Samples $(1 \mathrm{ml})$ were subjected to $10 \mathrm{~min}$ of darkness, followed by $10 \mathrm{~min}$ of red $(455 \mathrm{~nm})$ and blue $(630 \mathrm{~nm})$ illumination $\left(8.7 \mu \mathrm{E} / \mathrm{m}^{2} \mathrm{~s}\right)$. Oxygen production rate was calculated from the slope of oxygen evolution. Data was normalized and expressed as the oxygen produced by each microalga cell per second.

\subsection{PSOP Osmolarity and Viscosity}

The osmolality of PSOP containing different cell densities $\left(0,10^{6}\right.$, $10^{7}, 10^{8}$ and $10^{9}$ C. reinhardtii $\left./ \mathrm{ml}\right)$ was measured at RT using a cryoscopic osmometer (Osmomat 030, Gonotec). Viscosity was measured in a rheometer, using a $40 \mathrm{~mm}$ conical geometry in response to different shear rates (Discovery HR-2, TA Instruments). The gap between the sample and the geometry was set at $300 \mu \mathrm{m}$ and measurements were carried out at $28^{\circ} \mathrm{C}$.

\subsection{In vivo Toxicity Assay}

Zebrafish is a highly characterized and validated model for biomedical toxicity assays (Choi et al., 2021), thus ten larvae at 5 days post fertilization (Danio rerio, TAB5 strain) were obtained from our breeding colony and incubated at $28^{\circ} \mathrm{C}$, in 12-well plates, and exposed for $24 \mathrm{~h}$ to medium (E3, control) or PSOP with increasing cell densities of $C$. reinhardtii. In order to maintain larvae in optimal conditions, incubations were performed in a 14:10 light-dark photoperiod as we described before (Alvarez et al., 2018), and no additional illumination was provided to induce photosynthetic oxygen production. Then, survival was determined as the percentage of heart beating larvae. For morphological imaging, larvae were anesthetized by immersion in $4.2 \%(\mathrm{w} / \mathrm{v})$ tricaine (Sigma-Aldrich), euthanized by cold shock $\left(-20^{\circ} \mathrm{C}\right.$ for $\left.10 \mathrm{~min}\right)$, fixed in $4 \%$ paraformaldehyde and imaged with a stereoscope (Leica S6D).

\subsection{Metabolic Coupling Assay With Larvae and Rat Kidney Slices}

Twenty zebrafish larvae ( $5 \mathrm{dpf}$ ) contained in $1 \mathrm{ml}$ of RLM were added into the electrode chamber of the Oxygraph System
(Hansatech Instruments), and the oxygen evolution was measured for $5 \mathrm{~min}$ in darkness followed by $5 \mathrm{~min}$ of red $(455 \mathrm{~nm})$ and blue $(630 \mathrm{~nm})$ illumination $\left(8,7 \mu \mathrm{E} / \mathrm{m}^{2} \mathrm{~s}\right)$. Then, $200 \mu \mathrm{L}$ of PSOP containing $10^{8} \mathrm{C}$. reinhardtii/ml were added $\left(10^{6}\right.$ C. reinhardtii/larva), and the oxygen evolution was recorded for the next $10 \mathrm{~min}$ in the same lighting condition, followed by $10 \mathrm{~min}$ of darkness. Oxygen metabolic rate was calculated from the linear slope of oxygen concentration curve. For rat kidney slices the same setting was applied with slight modifications. Male Sprague-Dawley rats (250-400 g) were obtained from the animal facility of INTA, Universidad de Chile (Santiago, Chile). All animal experiments were performed according to protocols approved by the Ethics Committee of Pontificia Universidad Católica de Chile (180813015). Animals were anesthetized with ketamine $(90 \mathrm{mg} / \mathrm{kg}) / \mathrm{xylazine}(10 \mathrm{mg} / \mathrm{kg})$ i. p. and the kidneys were washed out of blood by perfusing $5 \mathrm{ml}$ warm RLM solution supplemented with $5 \% \quad(\mathrm{v} / \mathrm{w})$ dextran-70 through the abdominal aorta with a syringe. The left kidney was excised, cut in half, and mounted in a vibratome to obtain coronal slices. A single central slice $(500 \mu \mathrm{m}$ thick) was incorporated in the oxygraph chamber and incubated with $2 \mathrm{ml}$ of dextran supplemented RLM, and $100 \mu \mathrm{L}$ containing $2 \cdot 10^{7} \quad$ C. reinhardtii were added.

\subsection{Porcine Kidneys Procurement}

Female healthy pigs were selected by weight $(35-45 \mathrm{Kg}$ ) from our research breeder facility (CICAP-UC Pirque, Santiago, Chile). Animals were sedated with ketamine $(25 \mathrm{mg} / \mathrm{kg})$ and midazolam $(0.5 \mathrm{mg} / \mathrm{kg})$ and general anesthesia was maintained with $2 \%$ isoflurane and animals were connected to mechanical ventilation. Heparin (100-200 UI/kg) was administered to avoid coagulation during organs procurement. Kidneys were isolated and perfused with $500 \mathrm{ml}$ of Custodiol ${ }^{\circ}$ and kept at $4^{\circ} \mathrm{C}$ until experimental studies. Thereafter, pigs were euthanized with thiopental and potassium chloride. All the experiments were performed after the approval of our local ethical committees (approval No. 160126009).

\subsection{Microalgae Distribution After ex vivo Kidney Perfusion}

Porcine kidneys were manually perfused with $50 \mathrm{ml}$ of PSOP $(5 \times$ $10^{8}$ C. reinhardtii/ml) and submitted to macroscopic and histological analysis. For low magnification imaging, fresh organs were sliced with a surgical scalpel and pictures were taken using a stereoscope (Leica S6D). Then, biopsies were fixed in $4 \%$ paraformaldehyde, included in O.C.T. compound (4,583, Sakura), sliced $(30 \mu \mathrm{m})$, stained with $\mathrm{H} \& \mathrm{E}$ and imaged with Leica DM500 microscope.

\subsection{Machine for Dynamic Organ Perfusion}

A machine perfusion system was specially designed and manufactured for this study (Sky-Walkers SpA), which consisted of an organ receiving chamber, a volume reservoir, and a centrifugal pump (EC042B IDEA ${ }^{\circledR}$ Motor, Pittman) 

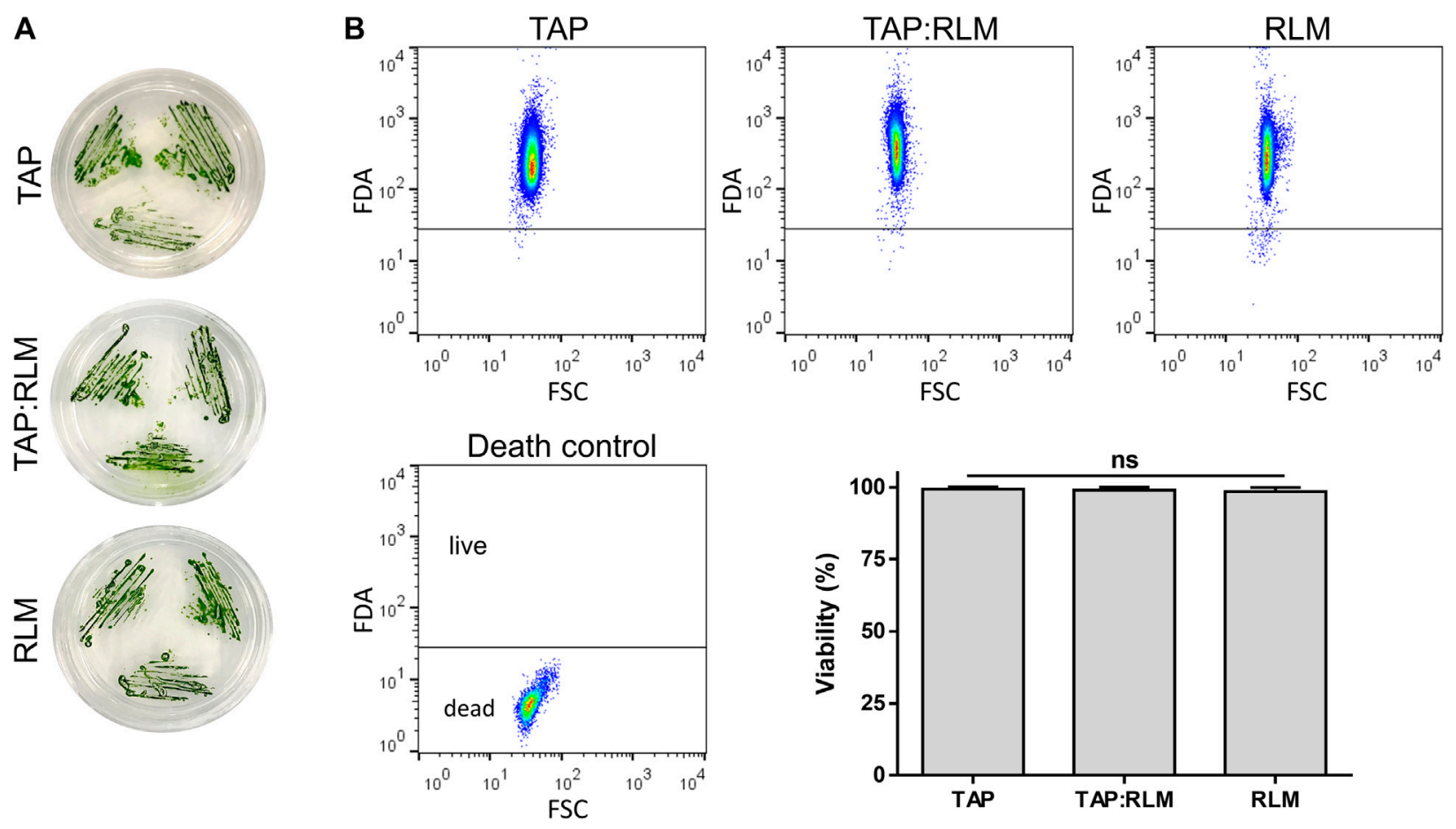

FIGURE 1 | Viability of C. reinhardtii in RLM. C. reinhardtii were incubated for $24 \mathrm{~h}$ in their culture media (TAP), a standard solution for organ preservation (RLM) or a mix of both in a 1:1 ratio (TAP:RLM). Viability of microalgae was not affected as shown by their growing capacity (A) and by flow cytometry (B). Data are expressed as mean $\pm S D ; N=3$; ns: non-significant (one-way ANOVA test).

connected to a reservoir, to carry out the flow to the renal artery. Pressure (TruWave disposable pressure transducers, Edwards Lifesciences) and flow sensors (Biomedicus TX50 Bio-probe flow transducer, Medtronics) were placed in the arterial line and a closed-loop pressure control was designed and manufactured to keep infusion pressure stable within physiological ranges $(70-80 \mathrm{mmHg})$.

\subsection{Dynamic Sub-normothermic ex vivo Perfusion of Porcine Kidneys}

Isolated kidneys were cannulated through aortal patch and connected to the perfusion machine described in section 2.11. Kidneys were perfused at RT for 30 min with continuous recirculating flow, using $1 \mathrm{~L}$ of PSOP at $5 \times 10^{7} \mathrm{C}$. reinhardtii/ml. Then, organs were flushed with RLM containing $5 \%$ dextran-70 (w/v) for $40 \mathrm{~min}$, without recirculation. Mean arterial pressure (MAP) and perfusion flow were recorded, and renal vascular resistance (RVR) was calculated as: $\mathrm{RVR}=\mathrm{MAP} /$ perfusion flow. Samples of PSOP before and during perfusion/flushing were diluted to $3 \times 10^{5}$ C. reinhardtii/ml, incubated with FDA and analyzed by flow cytometry as described in section 2.3. After perfusion and flushing, biopsies were fixed in $4 \%$ paraformaldehyde, included in paraffin, sliced $(4 \mu \mathrm{m})$ and stained with H\&E.

\subsection{Statistical Analysis}

All assays were performed in at least three independent experiments (unless specified). GraphPad Prism five software
(GraphPad Software) was used for statistical analyses. Statistical tests used are described in each result section.

\section{RESULTS}

\subsection{Functional Characterization of PSOP}

The first step to study the feasibility of using photosynthetic microalgae in a perfusable solution for ex vivo organ preservation was to evaluate whether the microalgae $C$. reinhardtii could survive in a standard perfusable physiological solution for organ preservation. Thus, microalgae were incubated for $24 \mathrm{~h}$ in RLM, standard algae medium (TAP) or a 1:1 mixture of both. Then, cell viability was assessed, both by evaluating their growing capacity in agar plates (Figure 1A) and by cell cytometry (Figure 1B). No significant differences were observed among groups, confirming that $C$. reinhardtii remain viable for at least $24 \mathrm{~h}$ in RLM. Then, possible morphological changes of $C$. reinhardtii induced by incubation in the RLM were also evaluated. As shown in Figure 2A, the general morphology and size of the microalgae did not vary, being further quantified by flow cytometry, where cell diameters of $7.5 \pm$ $0.3,7.4 \pm 0.5$ and $7.6 \pm 0.4 \mu \mathrm{m}$ were observed for RLM, TAP: RLM and RLM respectively (Figure 2B). Afterwards, oxygen production rates of RLM containing different densities of $C$. reinhardtii (PSOP) were characterized and compared. No significant differences in oxygen production were observed between 0 and $24 \mathrm{~h}$ of incubation in RLM, nor among the 

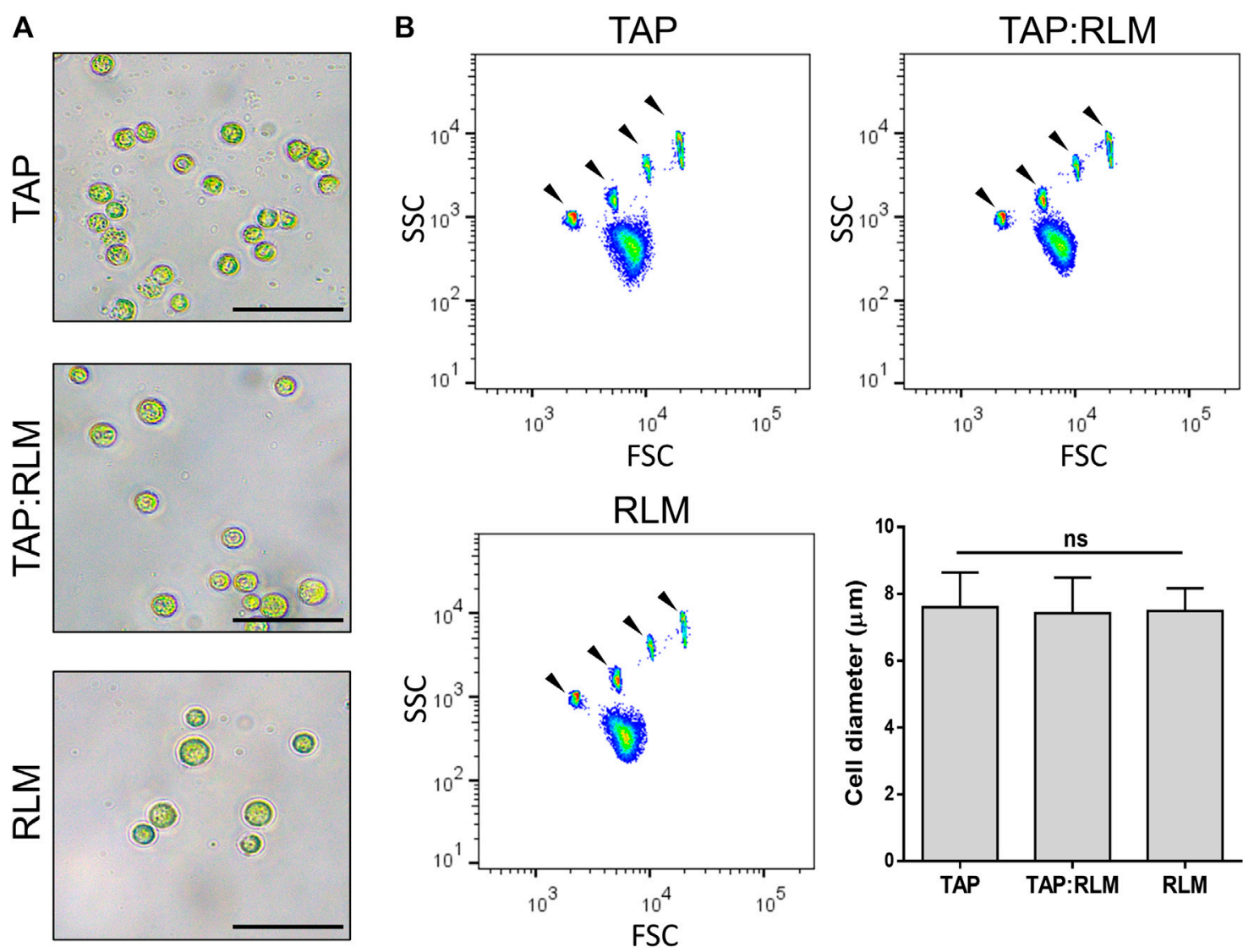

FIGURE 2 | Morphology of C. reinhardtii in RLM. C. reinhardtii were incubated for $24 \mathrm{~h}$ in their culture media (TAP), a standard solution for organ preservation (RLM) or a mix of both in a 1:1 ratio (TAP:RLM). Morphology $\mathbf{( A )}$ and size $\mathbf{( B )}$ of the microalgae were not affected by the media. Arrow heads indicate size marker beads of 4, 6 , 10 , and $15 \mu \mathrm{m}$ in diameter, from left to right. Scale bars represent $25 \mu \mathrm{m}$. Data are expressed as mean $\pm \mathrm{SD}$; $\mathrm{N}=3$; ns: non-significant (one-way ANOVA test).

different densities, except for the group containing $10^{6} \mathrm{C}$. reinhardtii/ml, which presented a higher production rate after $24 \mathrm{~h}$ of incubation (Figure 3A). Within the studied range, results show that single cell oxygen production was conserved at higher densities, remaining in the 30-40 (amol/cell s) range. Interestingly, no significant differences in cell number were observed after 24 of incubation, suggesting a decrease in the proliferation capacity of the microalgae in this particular experimental setting. Because maintaining osmotic pressure under physiological ranges is a fundamental requirement for perfusable solutions for organ preservation, osmolality was also quantified. As shown in Figure 3B, the presence of microalgae did not affect the osmolality of the PSOP, remaining in the range of $310 \mathrm{mOsm} / \mathrm{Kg}$, except for $10^{9} \mathrm{C}$. reinhardtii/ml where the osmolality increased significantly. The effect of the microalgae density in the rheological properties of the PSOP was also studied, measuring viscosity in response to increasing shear rates. Once again, up to densities of $10^{8} \mathrm{C}$. reinhardtii/ml, viscosity values were comparable to RLM, while at $10^{9}$ C. reinhardtii/ml, the solution presented significantly higher viscosity (Figure $3 \mathrm{C}$ ).

To evaluate potential toxic effects of the PSOP, zebrafish larvae were used as an in vivo vertebrate model for toxicity testing. After incubation, no obvious morphological changes, or signs of damage (such as edema formation and eye size reduction) were observed at different microalgae densities, except for the $10^{9}$ C. reinhardtii/ml, which induced a general curvature and twisting of the larvae (Figure 3D). In terms of viability, solutions containing up to $10^{7} \mathrm{C}$. reinhardtii/ml were non toxic for the larvae, while densities of $10^{8}$ and $10^{9} \mathrm{C}$. reinhardtii/ml, induced mild and severe mortality respectively (Figure $3 \mathrm{E}$ ).

\subsection{Metabolic Coupling Between PSOP and Animal Systems}

The capacity of PSOP to produce enough oxygen to support the metabolic requirements of an active biological system was evaluated. First, zebrafish larvae were placed in an Oxygraph chamber containing RLM, and the oxygen evolution was measured in the absence or presence of microalgae, as well as in the absence or presence of light (Figure 4A). In the absence of microalgae, the oxygen concentration decreased overtime, and the negative slope of the curve did not vary in the presence of light, indicating high oxygen consumption of larvae at this stage (Figures 4B,C, segment I and II). Afterwards, microalgae were added to the chamber, and the slope of the curve immediately 

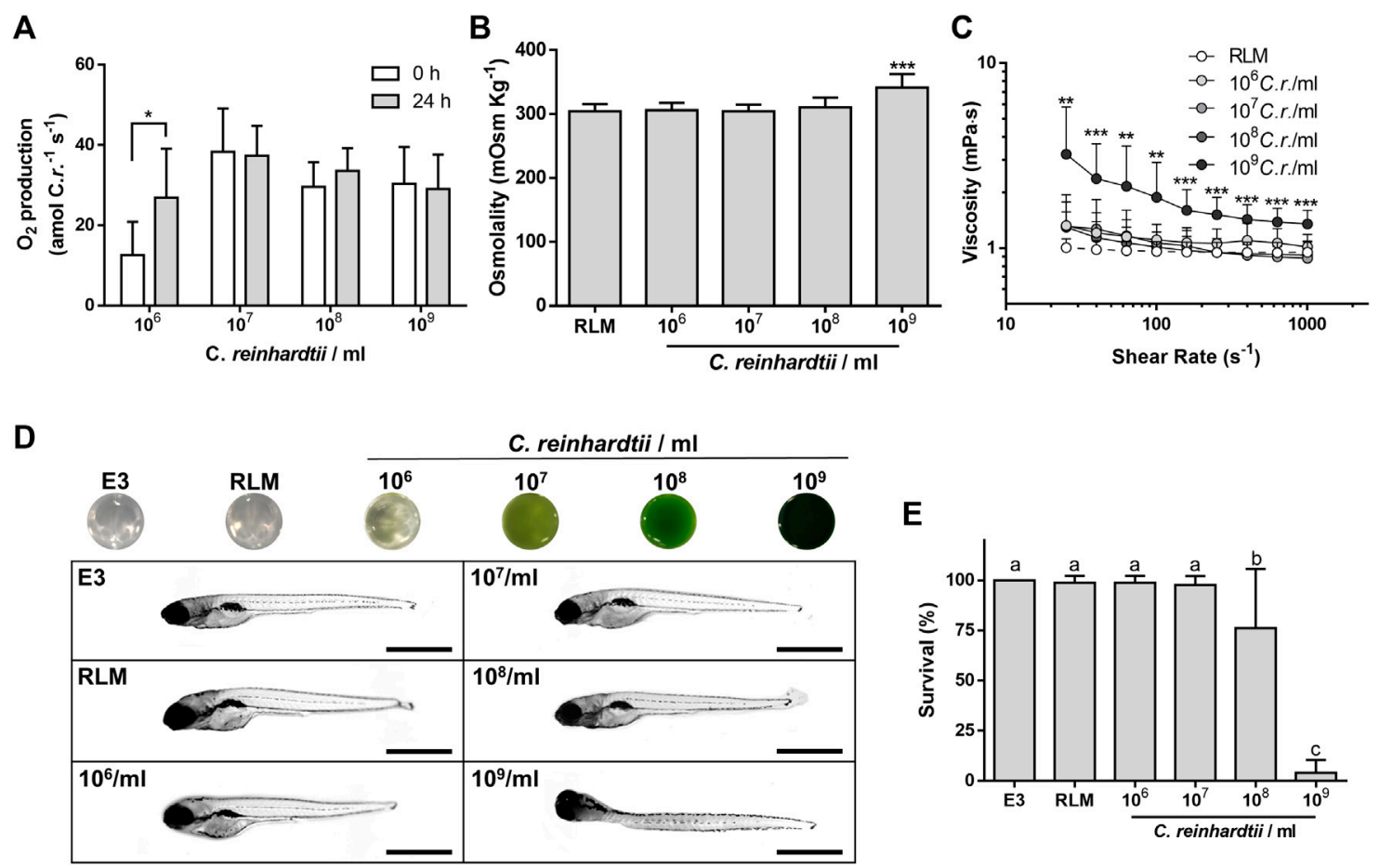

FIGURE 3|Characterization of the photosynthetic solution. Different densities of C. reinhardtii were added to a standard solution for organ preservation (RLM). The oxygen production rate of microalgae was maintained after $24 \mathrm{~h}$, except for $10^{6} \mathrm{C}$. reinhardtii/ml, which increased (A). Up to $10^{8} \mathrm{C}$. reinhardtii/ml, no significant differences were observed in the osmolality (B) and rheological properties of the solution (C). Zebrafish larvae were exposed for $24 \mathrm{~h}$ to photosynthetic solution containing different densities of microalgae, in the dark. Up to $10^{8} \mathrm{C}$. reinhardtii/ml larvae presented normal phenotypes compared to control [E3; (D)]. Mild and severe mortality were observed at $10^{8}$ and $10^{9} \mathrm{C}$. reinhardtii/ml, respectively (E). Scale bars represent $1 \mathrm{~mm}$ in D. Data are expressed as mean $\pm \mathrm{SD} ; \mathrm{N}=3,4 ;{ }^{*} p<0.05$, ${ }^{* \star *} p \leq 0.001$ (one-way ANOVA followed by Tukey's test in A; one-way ANOVA followed by Dunnett's test in B; two-way ANOVA followed by Sidak's test in C); different letters in E indicate significant differences with $p<0.05$ (one-way ANOVA followed by Tukey's test).

showed a positive slope, meaning that the oxygen production of PSOP exceeded the consumption rate of the larvae (Figures $4 B, C$, segment III). Finally, the light was turned off and a negative slope was observed again (Figures 4B,C, segment IV). To validate this data with a more relevant model for transplantation, the same setting was applied to fresh rat kidney slices, and similar results were obtained, as in the presence of light and microalgae, the negative slope of the curve was reverted in segment III, becoming nearly flat, indicating equal oxygen production and consumption; and finally turning negative again in segment IV upon turning off the light (Figures 4D,E).

\subsection{Characterization of ex vivo Perfused Porcine Kidneys}

In order to validate the PSOP in a clinically relevant model, isolated porcine kidneys were manually perfused, and renal tissue turned uniformly green, presenting an apparent slight swelling. Besides, no other macroscopic differences were observed among the non-perfused and perfused organs (Figure 5A). Then, fresh perfused tissues were sliced and observed under a stereoscope, clearly showing that microalgae reached the entire vascular territory of the organs. In contrast to calyxes and papilla, where the vascular density is lower, the cortex as well as the medullar pyramid exhibited an intense green color (Figure 5B). A more detailed localization of the microalgae was microscopically evaluated in cryosections, which showed their homogeneous presence through the entire vascular structures, including the globular distribution in the glomeruli with the afferent arteriole and the characteristic parallel line arrangement of the vessels in the renal medulla (Figure 5C).

To continue the PSOP validation, perfusion dynamics were evaluated in isolated porcine kidneys, which were connected to an organ perfusion machine prototype specially designed for this study (Figure 6A). This device has automatic flow control based on the sensed values to maintain physiological pressure throughout the procedure. The results showed a stable MAP of $75.5 \mathrm{mmHg}$ during perfusion and the following flushing step, confirming the reliability of the perfusion machine (Figure 6B). After the first $5 \mathrm{~min}$, the flow decreased from a mean of $56.7 \pm$ 3.3 to $26.7 \pm 8.8 \mathrm{ml} / \mathrm{min}$ by $15 \mathrm{~min}$, remaining constant until the flushing step with microalgae-free solution, where the flow gradually recovered and reached values up to $96.7 \pm 12.0 \mathrm{ml} /$ min (Figure 6C). Accordingly, during PSOP perfusion, calculated RVR increased from $1.26 \pm 0.05$ to a peak of 


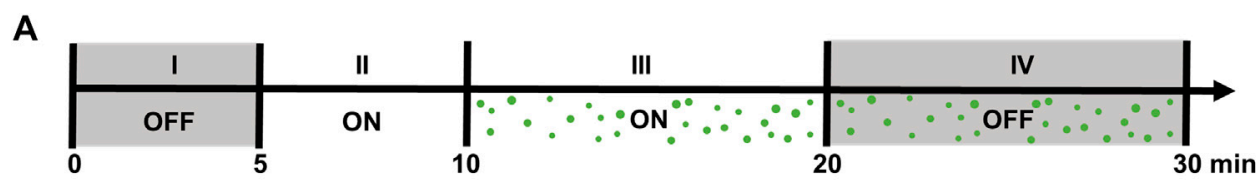

B

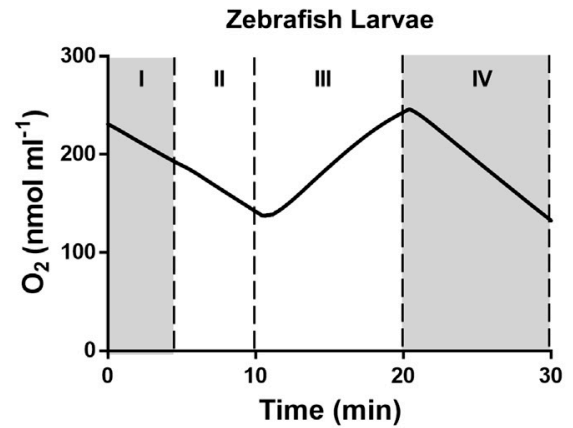

C

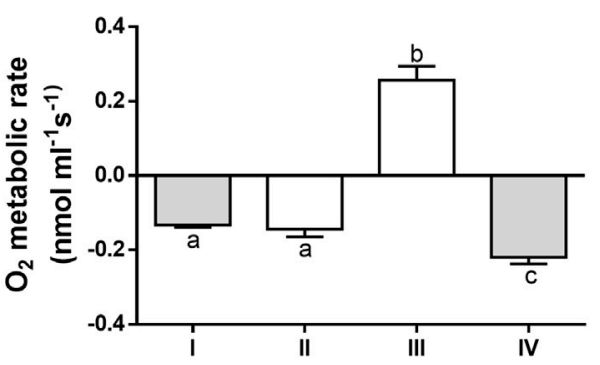

D

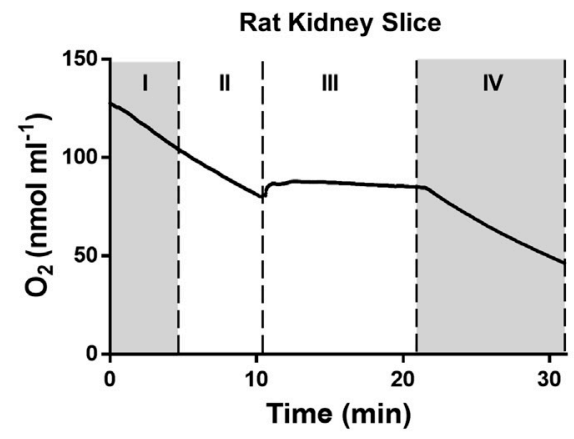

$\mathrm{E}$

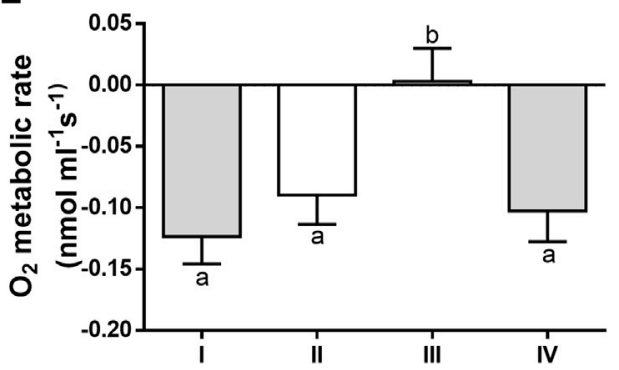

FIGURE 4 | Oxygenation capacity of the photosynthetic solution. Oxygen concentrations were measured for 5 min in darkness (I, OFF) or light (II, ON). Then, microalgae were incorporated and measurements were performed for $10 \mathrm{~min}$ in the presence (III, ON) or absence (IV, OFF) of light (A). In the absence of light or microalgae (I, II and IV) a negative slope of the curve was seen for both, zebrafish larvae (B,C) and kidney slices (D,E), while in the presence of light and microalgae, the slope became positive for the larvae and nearly flat for the slices. A representative curve is shown for each experiment (B-D) and their metabolic rates calculated from the slopes (C-E). Data are expressed as mean \pm SD; $N=3$; different letters in $\mathrm{C}$ and $\mathrm{E}$ indicate significant differences with $p<0.05$ (one-way ANOVA followed by Tukey's test).

$4.63 \pm 2.49 \mathrm{mmHg} \mathrm{min} / \mathrm{ml}$ at $16 \mathrm{~min}$, being then less stable with variations between $2.79 \pm 0.69$ and $4.31 \pm 1.89 \mathrm{mmHg} \mathrm{min} / \mathrm{ml}$. Thereafter, the initial RVR values were restored after $28 \mathrm{~min}$ of flushing (Figure 6D). Next, the effect of the perfusion itself in the integrity of the microalgae was studied. Here, samples were collected from the renal vein effluent and analyzed by flow cytometry. Interestingly, the perfusion process did not affect microalgae viability, remaining at about $80 \%$ during the perfusion $(83.4 \pm 6.7 \%)$ and the flushing step $(81.8 \pm 8.5 \%)$ (Figure 7A, upper). In addition to viability, the effect of perfusion in the morphology of the microalgae was also assessed, finding an almost complete overlapping between the microalgae populations obtained before and after perfusion (Figure 7A, lower). Finally, tissue integrity was analyzed after perfusion and flushing, and $\mathrm{H} \& \mathrm{E}$-stained paraffin sections showed the classic kidney architecture, represented by the cortex, containing renal corpuscles, proximal and distal tubules, and the medulla with collecting ducts and loops of Henle (Figure 7B). Overall, results show that glomeruli and tubules did not present signs of damage, as neither necrotic cells in Bowman's capsule nor blood cells or microalgae in Bowman's space were detected.

\section{DISCUSSION}

In this proof-of-concept study, we demonstrate the feasibility to incorporate photosynthetic microorganisms in organ perfusion solutions to provide an alternative intravascular source of oxygen to isolated organs. This concept is based on our previous research in implantable photosynthetic biomaterials (Hopfner et al., 2014; Schenck et al., 2015; Chávez et al., 2016; Chávez et al., 2021; Centeno-Cerdas et al., 2018), and could potentially generate a new physiological state of normoxic ischemia, where the lack of blood supply may not necessarily trigger hypoxia. The use of photosynthetic microorganisms as local oxygen factories may have significant advantages compared to the standard approaches. Among others, there is no need for additional carriers, and the local oxygen release kinetics could be easily controlled by the light intensity provided (Rühle et al., 2008). Additionally, this approach allows the generation of genetically modified photosynthetic organisms that, in addition to oxygen, could locally release fresh bioactive recombinant molecules as well (Chávez et al., 2020).

Ringer's lactate solution was chosen as the base to develop the PSOP because of its extended clinical use as physiological fluid 


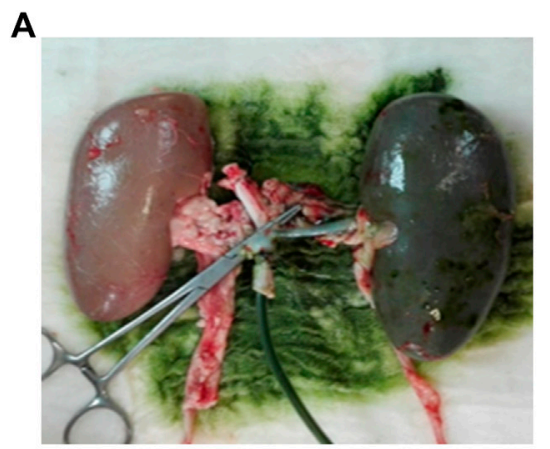

B
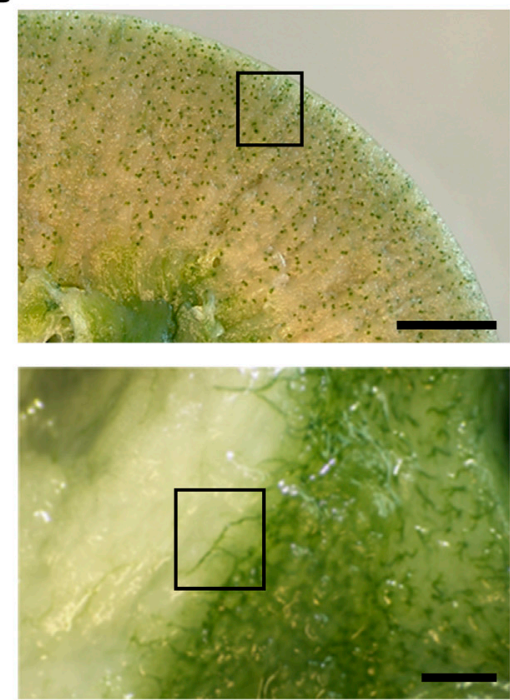
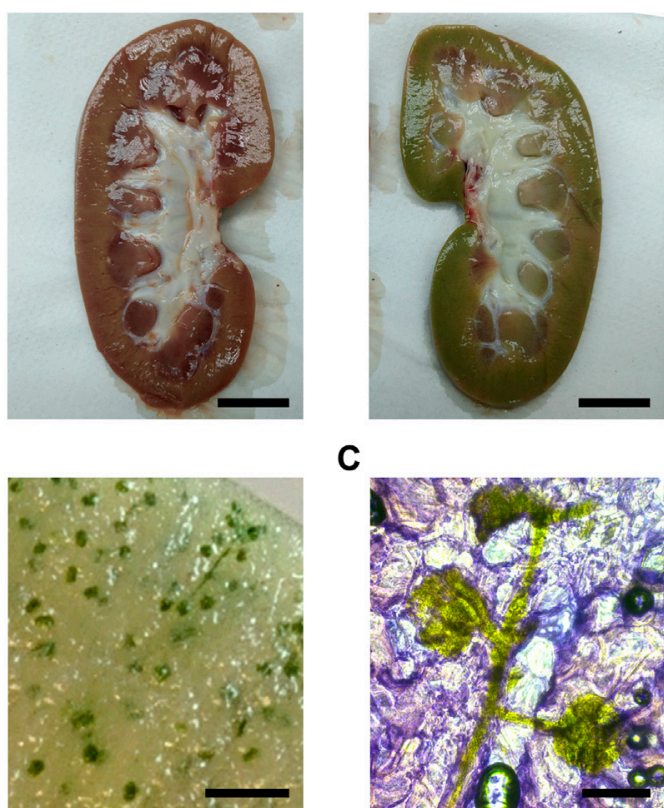

C
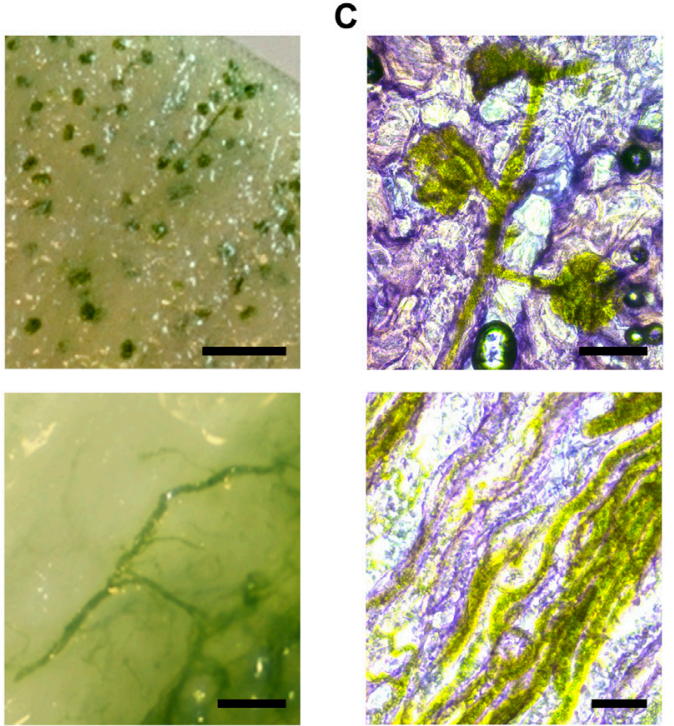

FIGURE 5 | Distribution of the photosynthetic solution in porcine kidney. Compared to controls, organs turned green after perfusion [(A), left and right]. Fresh slices show a vascular distribution of the solution in the renal cortex [(B), top] and medulla [(B), bottom]. Cryosections of perfused kidneys show the distribution of $C$. reinhardtii in glomeruli and afferent arteriole [(C), top] and medullar blood vessels and capillaries [(C), bottom]. Scale bar represents $2 \mathrm{~cm}$ in A, $5 \mathrm{~mm}$ in (B) (top, left), $1 \mathrm{~mm}$ in (B) (top, right) and (B) (bottom, left), $250 \mu \mathrm{m}$ in (B) (bottom, right), $100 \mu \mathrm{m}$ in (C).

(Singh and Davis, 2018; de Sousa et al., 2021). Mannitol was added as an impermeant agent to prevent cell swelling, one of the main requirements for preservation solutions (Nicholson and Hosgood, 2017). For kidney perfusion and oxygraphy of kidney slices, dextran-70 was also added to maintain the oncotic pressure and prevent tissue edema (Wang et al., 2004; Wilson et al., 2006). Although cell proliferation was not observed after $24 \mathrm{~h}$ of incubation in either medium (RLM and TAP), Ringer's lactate and mannitol solution (RLM) showed high biocompatibility with C. reinhardtii, without affecting microalgae viability and morphology, nor their photosynthetic capacity overtime. This result is somehow surprising because preservation solutions have to meet specific physicochemical requirements (Fuller et al., 2018) that differ from the optimal culture conditions of the microalgae. For instance, the osmolality of the microalgae medium (TAP) is around $64 \mathrm{mOsm} / \mathrm{Kg}$ (Komsic-Buchmann et al., 2012) while it is $305 \mathrm{mOsm} / \mathrm{Kg}$ for RLM, and previous studies have shown how an increase in osmotic stress can negatively affect cell growth and photosynthetic rates in $C$. reinhardtii (Neale and Melis, 1989). With exception of the highest microalgae density, all tested PSOP presented the same flowing behavior and dynamic viscosity values as RLM. It is worth noting that in all groups viscosity tended to decrease with higher shear rates (shear thinning), resembling blood behavior (Cherry and Eaton, 2013) and exhibiting appropriate rheological properties for organ perfusion. Moreover, at most concentrations of microalgae, the photosynthetic solution was biocompatible with zebrafish larvae. This toxicity model was chosen because is extensively used in several fields of research (Lieschke and Currie, 2007), and has been widely validated as a reliable model to test toxicity for biomedical application (Choi et al., 2021). In fact, the sensibility of this model shows that both, morphology and viability were significantly affected by incubations in PSOP at the highest density of microalgae, allowing to better define the potential clinical range for a safe microalgae perfusion procedure. This toxic effects at high microalgae densities could be due to several reasons including a high oxygen consumption of the microalgae under such insufficient illumination conditions, or due to issues related to the increased viscosity and osmolality of the PSOP. 
A

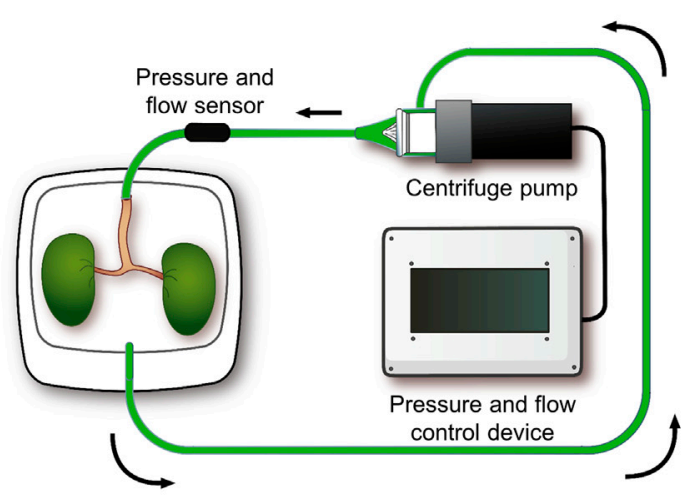

C

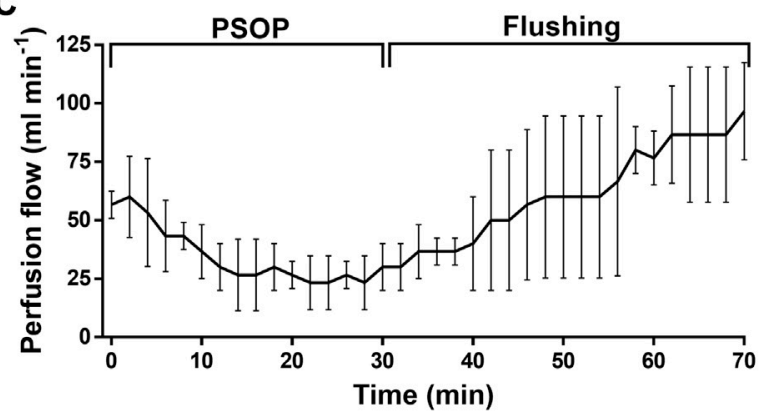

B

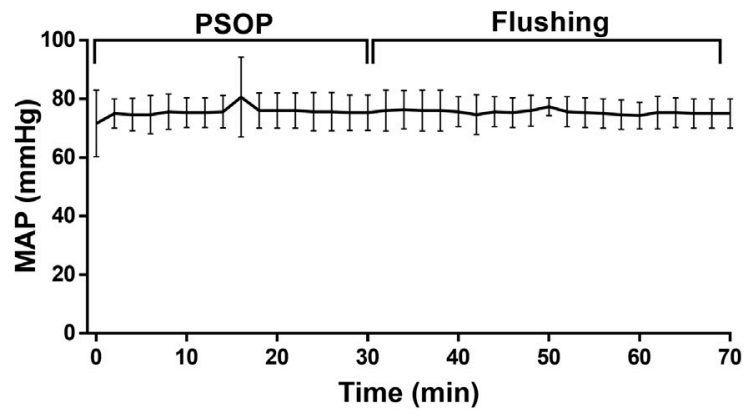

D

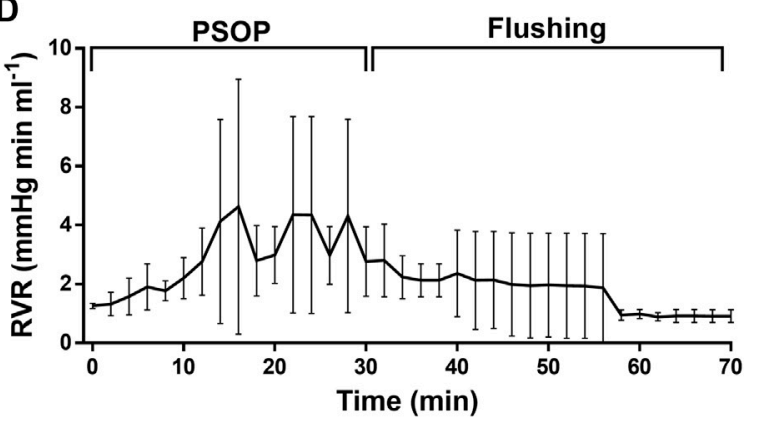

FIGURE 6|Dynamic perfusion of isolated porcine kidneys. Schematic representation of the ex vivo perfusion system, containing a pressure-flow controlling device, a centrifuge pump, and a container for the isolated organs (A). Vascular parameters were measured during the photosynthetic perfusion and the subsequent flushing step. Mean arterial pressure (MAP) was set to $70-80$ mmHg remaining stable during the entire procedure (B). Perfusion flow decreased during the PSOP perfusion (C), while renal vascular resistance (RVR) increased, recovering during the flushing step (D). Data are expressed as mean $\pm \mathrm{SEM}$; $\mathrm{N}=3$ in (B-D).

To evaluate the functionality of PSOP in a more clinical setting it is required to provide adequate illumination. Hence, an important limitation of this study is that, due to the lack of established technologies for optimal inner organ illumination, here we could not evaluate the functional effect of the PSOP in the oxygenation and further preservation of isolated organs, supporting the need of developing accurate illumination devices for this purpose. Nevertheless, we decided to evaluate the oxygenation capacity of PSOP in zebrafish larvae. In addition to the advantages described above, in contrast to other organisms, larvae are fully permeable, thus their entire gas interchange occurs by diffusion, allowing to quantify the metabolic interaction between the photosynthetic oxygen produced by the PSOP and the living animal tissues. Considering that five dpf larvae mass are approximately $0.5 \mathrm{mg}$ each (Barrionuevo et al., 2010), it follows that $10^{9}$ microalgae suspended in RLM would be sufficient to oxygenate $1 \mathrm{G}$ of tissue. However, because larval stages are highly hypermetabolic, this number of microalgae might be overestimated. Oxygen requirements of human cells can widely vary ranging from values below 1 to $350 \mathrm{amol} / \mathrm{cell} \mathrm{s}$ (Wagner et al., 2011). For example, human liver cells in culture are described to consume around $100 \mathrm{amol} / \mathrm{cell} \mathrm{s}$ (Wagner et al., 2011), which is promising when compared to our results showing that each microalga in the PSOP solution is capable to produce $30-40 \mathrm{amol} / \mathrm{cell} \mathrm{s}$ upon light exposure.
Therefore, a ratio of 3:1 microalgae to cell would be sufficient to ensure optimal tissue oxygenation for that cell type, especially relevant when considering that hepatocytes are roughly 500 times larger in volume (Marguerat and Bähler, 2012) and the metabolic oxygen requirements of tissues decrease by $50 \%$ at subnormothermic conditions (Karangwa et al., 2016). In fact, our results shows that $2 \times 10^{6}$ microalgae were sufficient to match the oxygen consumption of a $500 \mu \mathrm{m}$-thick rat kidney slice, weighting approximately $45 \mathrm{mg}$. Interestingly, similar results have been recently published, showing that a suspension of $C$. reinhardtii was also capable to oxygenate mouse brain slices in vitro (Voss et al., 2021). The setting described above strongly resembles the famous experiment performed by Joseph Priestley in 1772 where he showed that, when placed in a close compartment, a plant can provide enough oxygen to supply the metabolic requirements of a mouse (West, 2014). However, before the clinical translation of this approach, several issues need to be addressed especially in terms of the evaluation of safety and efficacy of this concept in human organs. Additionally, the composition of the PSOP described here will need to be optimized according to each particular clinical application, including its chemical composition, photosynthetic strain, and illumination setting.

As kidney represent the most frequent organ transplanted worldwide (WHO, 2020), we decided to initially evaluate the 

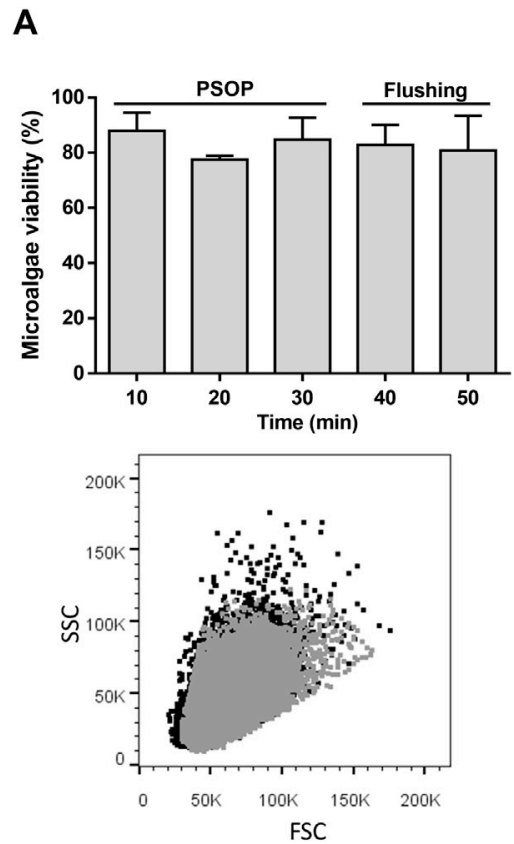

B

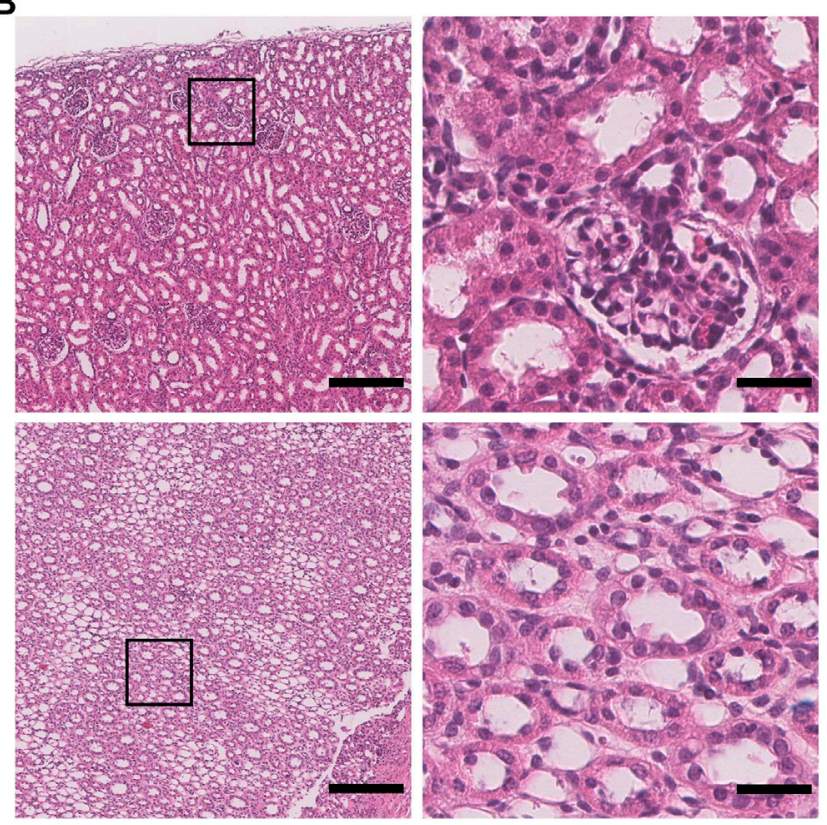

FIGURE 7 | Microalgae viability and renal tissue integrity after dynamic photosynthetic perfusion. Viability [(A), upper] and morphology [(A), lower] of the microalgae were not affected by the perfusion and the subsequent flushing step. H\&E-stained paraffin sections, shows a normal histological structure of porcine kidneys in cortex [(B); top] and medulla [(B); bottom] after perfusion. Black and grey dots in A (bottom) indicate microalgae samples of the solution obtained before and after 10 min of perfusion, respectively, showing an almost complete overlapping of the signal where gray dots masked the microalgae population represented by black dots. Scale bar represents $200 \mu \mathrm{m}$ [(B), left] and $30 \mu \mathrm{m}[(\mathbf{B})$, right]. Data are expressed as mean $\pm \mathrm{SD} ; \mathrm{N}=2$ in $\mathrm{A}$.

perfusion dynamic of PSOP in isolated porcine kidneys. Additionally, due to the kidney's intrinsic complexity, this approach allows to test the solution under highly challenging conditions, providing important information about its ex vivo rheological properties, and the effect of this perfusable solution in the different renal vascular domains. Interestingly, C. reinhardtii could reach even the smallest capillaries of porcine kidneys and survive the circulation process without damaging the general tissue architecture of the organs. However, a transient increase in the vascular resistance to flow was observed during $5 \times 10^{7} \mathrm{cell} / \mathrm{ml}$ PSOP perfusion, which can be due mostly to a simple increase in fluid viscosity as inferred from the rheological characterization (Figure 3C). This analysis, together with the recovery of resistance upon flushing, minimized the probability that reversible vascular occlusion could occur in this setting.

To optimize the approach presented here, further research should explore the immense biodiversity of photosynthetic microorganisms to find the most appropriate cell that may fit to every different organ preservation setting. This is valid for cell size, and other key features such as optimal temperature, osmolality, oxygen production, or illumination requirements. For instance, photosynthetic microorganisms range widely in size, from Ostreococcus tauri with less than $1 \mu \mathrm{m}$ of diameter, being the smallest free-living eukaryote known; and some other species such as Entemoneis kufferati and Synechococcus lividus that live in environments from 0 up to 72 Celsius degrees, respectively (Varshney et al., 2015).

As conclusion, this work provides the first evidence to support the use of photosynthetic perfusable solutions for organ preservation, representing another important step towards human photosynthesis and its potential therapeutic applications.

\section{DATA AVAILABILITY STATEMENT}

All data associated with this study are in the paper, and can be shared with approved outside collaborators under a materials transfer agreement; requests should be sent to JTE jte@uc.cl or RR, rareboll@uc.cl.

\section{ETHICS STATEMENT}

The animal study was reviewed and approved by the Local ethical committees (approval Nr. 160126009). Male Sprague-Dawley rats (250-400 g) were obtained from the animal facility of INTA, Universidad de Chile (Santiago, Chile). All animal experiments were performed according to protocols approved by the Ethics Committee of Pontificia Universidad Católica de Chile (180813015). 


\section{AUTHOR CONTRIBUTIONS}

JTE, RR, and MPB conceived the study and supervised the experiments. VV, RC-O, and $\mathrm{CE}$ planned, performed and interpreted the in vitro experiments. RR, SR, CM, RE, and DN planned, performed and interpreted porcine experiments. CG and RR designed, built and tested the perfusion machine. JTE, $\mathrm{VV}, \mathrm{RR}, \mathrm{RC}-\mathrm{O}$, and MPB wrote the paper. All authors contributed, reviewed and approved the final version of this manuscript.

\section{FUNDING}

This project was financed by CORFO grant 18COTE89629 and 18PIDE98887, National Agency for Research and Development (ANID) PhD Scholarship Program grants 2018-21181181 and

\section{REFERENCES}

Aburawi, M. M., Fontan, F. M., Karimian, N., Eymard, C., Cronin, S., Pendexter, C., et al. (2019). Synthetic Hemoglobin-based Oxygen Carriers Are an Acceptable Alternative for Packed Red Blood Cells in Normothermic Kidney Perfusion. Am. J. Transpl. 19, 2814-2824. doi:10.1111/ajt.15375

Alvarez, M., Chávez, M. N., Miranda, M., Aedo, G., Allende, M. L., and Egaña, J. T. (2018). A Novel In Vivo Model to Study Impaired Tissue Regeneration Mediated by Cigarette Smoke. Sci. Rep. 8, 1-12. doi:10.1038/s41598-01828687-1

Barrionuevo, W., Fernandes, M., and Rocha, O. (2010). Aerobic and Anaerobic Metabolism for the Zebrafish, Danio rerio, Reared under Normoxic and Hypoxic Conditions and Exposed to Acute Hypoxia during Development. Braz. J. Biol. 70, 425-434. doi:10.1590/s151969842010000200027

Bhattacharjee, R. N., Patel, S. V. B., Sun, Q., Jiang, L., Richard-Mohamed, M., Ruthirakanthan, A., et al. (2020). Renal protection against Ischemia Reperfusion Injury: Hemoglobin-Based Oxygen Carrier-201 versus Blood as an Oxygen Carrier in Ex Vivo Subnormothermic Machine Perfusion. Transplantation 104, 482-489. doi:10.1097/TP.0000000000002967

Bodewes, S. B., van Leeuwen, O. B., Thorne, A. M., Lascaris, B., Ubbink, R., Lisman, T., et al. (2021). Oxygen Transport during Ex Situ Machine Perfusion of Donor Livers Using Red Blood Cells or Artificial Oxygen Carriers. Ijms 22, 235. doi:10.3390/ijms22010235

Centeno-Cerdas, C., Jarquín-Cordero, M., Chávez, M. N., Hopfner, U., Holmes, C., Schmauss, D., et al. (2018). Development of Photosynthetic Sutures for the Local Delivery of Oxygen and Recombinant Growth Factors in Wounds. Acta Biomater. 81, 184-194. doi:10.1016/j.actbio.2018.09.060

Chávez, M. N., Fuchs, B., Moellhoff, N., Hofmann, D., Zhang, L., Selão, T. T., et al. (2021). Use of Photosynthetic Transgenic Cyanobacteria to Promote Lymphangiogenesis in Scaffolds for Dermal Regeneration. Acta Biomater. 126, 132-143. doi:10.1016/j.actbio.2021.03.033

Chávez, M. N., Moellhoff, N., Schenck, T. L., Egaña, J. T., and Nickelsen, J. (2020). Photosymbiosis for Biomedical Applications. Front. Bioeng. Biotechnol. 8. doi:10.3389/fbioe.2020.577204

Chávez, M. N., Schenck, T. L., Hopfner, U., Centeno-Cerdas, C., Somlai-Schweiger, I., Schwarz, C., et al. (2016). Towards Autotrophic Tissue Engineering: Photosynthetic Gene Therapy for Regeneration. Biomaterials 75, 25-36. doi:10.1016/j.biomaterials.2015.10.014

Chen, H., Cheng, Y., Tian, J., Yang, P., Zhang, X., Chen, Y., et al. (2020). Dissolved Oxygen from Microalgae-Gel Patch Promotes Chronic Wound Healing in Diabetes. Sci. Adv. 6. doi:10.1126/sciadv.aba4311

Cherry, E. M., and Eaton, J. K. (2013). Shear Thinning Effects on Blood Flow in Straight and Curved Tubes. Phys. Fluids 25, 073104. doi:10.1063/1.4816369
2015-21151013, ANID PAI program grants 7818T20003 and T78191E003 and FONDECYT grant 1200280.

\section{ACKNOWLEDGMENTS}

We thank Peter von Dassow and Jorge Ibáñez (Pontificia Universidad Católica de Chile), Yessia Hidalgo (Cells for Cells, Consorcio Regenero) and Flow Cytometry Core UC (Pontificia Universidad Católica de Chile) for their support with flow cytometry; Sebastián San Martín (Universidad de Valparaíso) for the histological analysis; Javier Enrione (Universidad de los Andes) for his support in rheology; Claudio Araya (Universidad Austral de Chile) and Miguel Miranda (Pontificia Universidad Católica de Chile) for their support with zebrafish larvae; Mario Faúndez (Pontificia Universidad Católica de Chile) for his support with oxygraphy.

Choi, T.-Y., Choi, T.-I., Lee, Y.-R., Choe, S.-K., and Kim, C.-H. (2021). Zebrafish as an Animal Model for Biomedical Research. Exp. Mol. Med. 53, 310-317. doi:10.1038/s12276-021-00571-5

Cohen, J. E., Goldstone, A. B., Paulsen, M. J., Shudo, Y., Steele, A. N., Edwards, B. B., et al. (2017). An Innovative Biologic System for Photon-Powered Myocardium in the Ischemic Heart. Sci. Adv. 3, e1603078. doi:10.1126/ sciadv. 1603078

de Sousa, S. G., Nascimento da Silva, G. V., Costa Rodrigues, A. M., Meireles Fernandes da Silva, T. M., Costa, F. C., Freitas Teixeira da Silva, A., et al. (2021). Organ Preservation Solutions in Transplantation: A Literature Review. Exp. Clin. Transpl. 19, 511-521. doi:10.6002/ect.2020.0506

de Vries, R. J., Yarmush, M., Uygun, K., Hospital, M. G., Medical, H., and Brunswick, N. (2019). Systems Engineering the Organ Preservation Process for Transplantation. Curr. Opin. Biotechnol. 58, 192-201. doi:10.1016/ j.copbio.2019.05.015

Eltzschig, H. K., and Eckle, T. (2011). Ischemia and Reperfusion-From Mechanism to Translation. Nat. Med. 17, 1391-1401. doi:10.1038/nm.2507

Evron, Y., Zimermann, B., Ludwig, B., Barkai, U., Colton, C., Weir, G., et al. (2015) Oxygen Supply by Photosynthesis to an Implantable Islet Cell Device. Horm. Metab. Res. 47, 24-30. doi:10.1055/s-0034-1394375

Fuller, B., Froghi, F., and Davidson, B. (2018). Organ Preservation Solutions. Curr. Opin. Organ. Transpl. 23, 361-368. doi:10.1097/MOT.0000000000000525

Giwa, S., Lewis, J. K., Alvarez, L., Langer, R., Roth, A. E., Church, G. M., et al. (2017). The Promise of Organ and Tissue Preservation to Transform Medicine. Nat. Biotechnol. 35, 530-542. doi:10.1038/nbt.3889

Gkoumassi, E., Dijkstra-Tiekstra, M. J., Hoentjen, D., and De Wildt-Eggen, J. (2012). Hemolysis of Red Blood Cells during Processing and Storage. Transfusion 52, 489-492. doi:10.1111/j.1537-2995.2011.03298.x

Haraguchi, Y., Kagawa, Y., Sakaguchi, K., Matsuura, K., Shimizu, T., and Okano, T. (2017). Thicker Three-Dimensional Tissue from a "symbiotic Recycling System" Combining Mammalian Cells and Algae. Sci. Rep. 7, 1-10. doi: $10.1038 /$ srep 41594

Harris, E. H. (2009). The Chlamydomonas Sourcebook: Introduction to Chlamydomonas and its Laboratory Use. Second. Elsevier.

Hopfner, U., Schenck, T.-L., Chávez, M.-N., Machens, H.-G., Bohne, A.-V., Nickelsen, J., et al. (2014). Development of Photosynthetic Biomaterials for In Vitro Tissue Engineering. Acta Biomater. 10, 2712-2717. doi:10.1016/ j.actbio.2013.12.055

Hosgood, S. A., Nicholson, H. F. L., and Nicholson, M. L. (2012). Oxygenated Kidney Preservation Techniques. Transplantation 93, 455-459. doi:10.1097/ TP.0b013e3182412b34

Huo, M., Wang, L., Zhang, L., Wei, C., Chen, Y., and Shi, J. (2020). Photosynthetic Tumor Oxygenation by Photosensitizer-Containing Cyanobacteria for Enhanced Photodynamic Therapy. Angew. Chem. 132, 1922-1929. doi:10.1002/ange.201912824 
H. Wilson, C., R. Brook, N., and Talbot, D. (2006). Preservation Solutions for Solid Organ Transplantation. Mrmc 6, 1081-1090. doi:10.2174/138955706778560148

Jägers, J., Wrobeln, A., and Ferenz, K. B. (2021). Perfluorocarbon-based Oxygen Carriers: from Physics to Physiology. Pflugers Arch. - Eur. J. Physiol. 473, 139-150. doi:10.1007/s00424-020-02482-2

Jamers, A., Lenjou, M., Deraedt, P., Bockstaele, D. V., Blust, R., and Coen, W. d. (2009). Flow Cytometric Analysis of the Cadmium-Exposed green algaChlamydomonas reinhardtii(Chlorophyceae). Eur. J. Phycology 44, 541-550. doi:10.1080/09670260903118214

Karangwa, S. A., Dutkowski, P., Fontes, P., Friend, P. J., Guarrera, J. V., Markmann, J. F., et al. (2016). Machine Perfusion of Donor Livers for Transplantation: A Proposal for Standardized Nomenclature and Reporting Guidelines. Am. J. Transpl. 16, 2932-2942. doi:10.1111/ajt.13843

Komsic-Buchmann, K., Stephan, L. M., and Becker, B. (2012). The SEC6 Protein Is Required for Function of the Contractile Vacuole in Chlamydomonas Reinhardtii. J. Cel Sci. 125, 2885-2895. doi:10.1242/jcs.099184

Le Meur, Y., Badet, L., Essig, M., Thierry, A., Büchler, M., Drouin, S., et al. (2020). First-in-human Use of a marine Oxygen Carrier (M101) for Organ Preservation: A Safety and Proof-of-principle Study. Am. J. Transpl. 20, 1729-1738. doi:10.1111/ajt.15798

Lieschke, G. J., and Currie, P. D. (2007). Animal Models of Human Disease: Zebrafish Swim into View. Nat. Rev. Genet. 8, 353-367. doi:10.1038/nrg2091

Liu, L., He, H., Luo, Z., Zhou, H., Liang, R., Pan, H., et al. (2020). In Situ Photocatalyzed Oxygen Generation with Photosynthetic Bacteria to Enable Robust Immunogenic Photodynamic Therapy in Triple-Negative Breast Cancer. Adv. Funct. Mater. 30, 1910176. doi:10.1002/adfm.201910176

Malchesky, P. S. (2017). Artificial Oxygen Carriers. Artif. Organs 41, 311. doi:10.1111/aor.12946

Marguerat, S., and Bähler, J. (2012). Coordinating Genome Expression with Cell Size. Trends Genet. 28, 560-565. doi:10.1016/j.tig.2012.07.003

Neale, P. J., and Melis, A. (1989). Salinity-stress Enhances Photoinhibition of Photosynthesis in Chlamydomonas Reinhardtii. J. Plant Physiol. 134, 619-622. doi:10.1016/S0176-1617(89)80158-0

Neupert, J., Karcher, D., and Bock, R. (2009). Generation ofChlamydomonasstrains that Efficiently Express Nuclear Transgenes. Plant J. 57, 1140-1150. doi:10.1111/j.1365-313X.2008.03746.x

Nicholson, M. L., and Hosgood, S. A. (2017). Organ Retrieval and Preservation. Surgery (Oxford) 35, 346-352. Obfervations, X. I. X., and Prieftley, J. I XIX. doi:10.1016/j.mpsur.2017.04.012

Obaíd, M. L., Camacho, J. P., Brenet, M., Corrales-Orovio, R., Carvajal, F., and Martorell, X. (2021). A First in Human Trial Implanting Microalgae Shows Safety of Photosynthetic Therapy for the Effective Treatment of Full Thickness Skin Wounds. Front. Med. 8, 772324. doi:10.3389/ fmed.2021.772324

Qiao, Y., Yang, F., Xie, T., Du, Z., Zhong, D., Qi, Y., et al. (2020). Engineered Algae: A Novel Oxygen-Generating System for Effective Treatment of Hypoxic Cancer. Sci. Adv. 6. doi:10.1126/sciadv.aba5996

Rühle, T., Hemschemeier, A., Melis, A., and Happe, T. (2008). A Novel Screening Protocol for the Isolation of Hydrogen Producing Chlamydomonas Reinhardtiistrains. BMC Plant Biol. 8. doi:10.1186/1471-2229-8-107

Schenck, T. L., Hopfner, U., Chávez, M. N., Machens, H.-G., Somlai-Schweiger, I., Giunta, R. E., et al. (2015). Photosynthetic Biomaterials: A Pathway towards Autotrophic Tissue Engineering. Acta Biomater. 15, 39-47. doi:10.1016/ j.actbio.2014.12.012

Singh, S., and Davis, D. (2018). "Ringer' S Lactate," in StatPearls. Treasure Island, FL: StatPearls Publishing [Internet], 12-15.

Slegtenhorst, B. R., Dor, F. J. M. F., Rodriguez, H., Voskuil, F. J., and Tullius, S. G. (2014). Ischemia/Reperfusion Injury and its Consequences on Immunity and Inflammation. Curr. Transpl. Rep. 1, 147-154. doi:10.1007/s40472-014-0017-6
Soo, E., Marsh, C., Steiner, R., Stocks, L., and McKay, D. B. (2020). Optimizing Organs for Transplantation; Advancements in Perfusion and Preservation Methods. Transplant. Rev. 34, 100514. doi:10.1016/j.trre.2019.100514

Tatum, R., O'Malley, T. J., Bodzin, A. S., and Tchantchaleishvili, V. (2021). Machine Perfusion of Donor Organs for Transplantation. Artif. Organs 45, 682-695. doi:10.1111/aor.13894

Varney, J., Rivera, A., Dong, V., Tieu, P., Zia, S., and Huy, N. T. (2021). Mini-review on the Properties and Possible Applications of Therapeutic Oxygen Carrier HemarinaM101. Transfus. Apher. Sci. 60, 103016. doi:10.1016/j.transci.2020.103016

Varshney, P., Mikulic, P., Vonshak, A., Beardall, J., and Wangikar, P. P. (2015). Extremophilic Micro-algae and Their Potential Contribution in Biotechnology. Bioresour. Tech. 184, 363-372. doi:10.1016/j.biortech.2014.11.040

Voss, L. J., Plouviez, M., and Whittle, N. (2021). Microalgae-based Photosynthetic Strategy for Oxygenating Avascularised Mouse Brain Tissue - an In Vitro Proof of Concept Study. Brain Res. 1768, 147585. doi:10.1016/j.brainres.2021.147585

Wagner, B. A., Venkataraman, S., and Buettner, G. R. (2011). The Rate of Oxygen Utilization by Cells. Free Radic. Biol. Med. 51, 700-712. doi:10.1016/ j.freeradbiomed.2011.05.024

Wang, J., Nation, R. L., Evans, A. M., and Cox, S. (2004). Isolated Rat Kidney Perfused with Dextran and Bovine Serum Albumin: A Stable Model for Investigating Renal Drug Handling. J. Pharmacol. Toxicol. Methods 49, 105-113. doi:10.1016/j.vascn.2003.11.001

West, J. B. (2014). Joseph Priestley, Oxygen, and the Enlightenment. Am. J. Physiology-Lung Cell Mol. Physiol. 306, L111-L119. doi:10.1152/ ajplung.00310.2013

WHO (2020). International Report on Organ Donation and Transplantation Activities. Available at: http://www.transplant-observatory.org/global-report2018/(Accessed December 29, 2020)

Xu, J., Buchwald, J. E., and Martins, P. N. (2020). Review of Current Machine Perfusion Therapeutics for Organ Preservation. Transplantation 104, 1792-1803. doi:10.1097/TP.0000000000003295

Yamaoka, I., Kikuchi, T., Arata, T., and Kobayashi, E. (2012). Organ Preservation Using a Photosynthetic Solution. Transpl. Res. 1, 2. doi:10.1186/2047-1440-1-2

Zhao, H., Alam, A., Soo, A. P., George, A. J. T., and Ma, D. (2018). IschemiaReperfusion Injury Reduces Long Term Renal Graft Survival: Mechanism and beyond. EBioMedicine 28, 31-42. doi:10.1016/j.ebiom.2018.01.025

Conflict of Interest: Author CG was employed by company Sky-Walker SpA.

Competing Interests: JTE is CSO and co-founder of SymbiOx Inc., a start-up company that owns IP in the field of this work. Thanks to an R and D grant provided by the Chilean Ministry of Economics (CORFO), during the conduct of this project, DN, RE and RC-O were full-time employees of SymbiOx Inc. All other authors declare that they have no competing interests.

Publisher's Note: All claims expressed in this article are solely those of the authors and do not necessarily represent those of their affiliated organizations, or those of the publisher, the editors and the reviewers. Any product that may be evaluated in this article, or claim that may be made by its manufacturer, is not guaranteed or endorsed by the publisher.

Copyright (๑) 2021 Veloso-Giménez, Escamilla, Necuñir, Corrales-Orovio, Riveros, Marino, Ehrenfeld, Guzmán, Boric, Rebolledo and Egaña. This is an open-access article distributed under the terms of the Creative Commons Attribution License (CC $B Y)$. The use, distribution or reproduction in other forums is permitted, provided the original author(s) and the copyright owner(s) are credited and that the original publication in this journal is cited, in accordance with accepted academic practice. No use, distribution or reproduction is permitted which does not comply with these terms. 